\title{
Technique of neo-pharynx reconstruction with supra-clavicular artery island flap in a case of pharyngeal stricture
}

\author{
Viresh Arora $^{1 *}$, Bhushan Kathuria², Madhuri Arora ${ }^{3}$
}

\author{
${ }^{1}$ Department of ENT, United Lincolnshire Hospitals Trust, UK \\ ${ }^{2}$ Department of ENT, Kainos Super Specialty Hospital, Rohtak, Haryana, India \\ ${ }^{3}$ Department of ENT, Pandit Bhagwat Dayal Sharma Post Graduate Institute of Medical Sciences, Rohtak, Haryana, \\ India
}

Received: 28 October 2020

Revised: 11 December 2020

Accepted: 15 December 2020

\author{
*Correspondence: \\ Dr. Viresh Arora, \\ E-mail: draroraent@gmail.com
}

Copyright: (C) the author(s), publisher and licensee Medip Academy. This is an open-access article distributed under the terms of the Creative Commons Attribution Non-Commercial License, which permits unrestricted non-commercial use, distribution, and reproduction in any medium, provided the original work is properly cited.

\begin{abstract}
Pharyngeal stenosis frequently occurs after laryngectomy or laryngo-pharyngectomy, more commonly in patients complicated with a post-operative pharyngo-cutaneous fistula. Oral feeding becomes challenging, restricted to liquids. In such cases, dilatations can be futile if there is a complete stricture necessiating surgery. We present a case of 72 year-old man who underwent total laryngectomy with partial pharyngectomy with pectoral major myocutaneous flap reconstruction for second primary of hypopharyngeal T3N0M0, after primary laryngeal T1N0M0 treated with RT presented with nearly $4 \mathrm{~cm}$ pharyngeal stricture causing him absolute dysphagia. Multiple dilations failed to create adequate passage, pharyngeal stricture was then excised, and a neopharynx was constructed with supraclavicular artery island flap. The outcome was uneventful with restoration of normal pharyngeal permeability and swallowing by $12^{\text {th }}$ post-operative day. Post-operative follow-up after 8 months remained satisfactory. Resection of limited height pharyngeal stenosis can be successfully performed as end-to-end anastomosis whereas reconstruction of a long segment pharyngeal stricture utilizing a loco-regional flap gives satisfactory outcome.
\end{abstract}

Keywords: Hypopharyngeal malignancy, Post-laryngectomy pharyngeal stricture, Circumferential pharyngeal defect, Supraclavicular artery island flap

\section{INTRODUCTION}

Reconstructing a circumferential hypopharyngeal defect is fraught with challenges. The primary objective not only involves restoration of the resected pharyngeal conduit for progression of food, but also maintaining the patency of the anastomosis to facilitate proper swallowing. ${ }^{1}$ Diagnosis, objective evaluation and treatment of pharyngeal stenosis with adequate outcome is an arduous task for the operating surgeon. ${ }^{2}$ Multiple options are available for the pharyngolaryngeal reconstruction such as inferior island trapezius myocutaneous flap, radial forearm free flap (RFFF), gastric pull-up and jejunal free flap; however, opinions differ amongst surgeons for the best outcome. Supra-clavicular artery island flap (SCAIF) has gained popularity in the head and neck reconstructive surgeries and is being used extensively. ${ }^{3}$ SCAIF has been extensively used as a flap; we would report this case as a first for pharyngeal defect reconstruction following resection of pharyngeal stricture, and flap is a valuable tool for head and neck defect reconstruction because of this versatility.

\section{CASE REPORT}

A 72-year-old male presented to the ear, nose and throat outpatient department (ENT OPD) with progressive dyspnea and loss of voice due to non-functional trachea- 
esophageal prosthesis and absolute dysphagia for 18 months. The patient had been a chronic smoker for 40 pack years with nearly 14 units of alcohol/week for last 40 years. Prior medical history revealed partial gastrectomy for perforated peptic ulcer 30 years before. He was diagnosed with carcinoma larynx (T1N0M0) and was treated with radiotherapy following which he remained symptom free for nearly 8 years. 2 years ago he developed a $2^{\text {nd }}$ primary tumour on the left side of his hypopharynx and T3N0M0 stage was confirmed. The patient opted for CRT instead of surgery and received 55 Gy radiation with 2 cycles of cisplatin and 5-fluorouracil. There was incomplete resolution of the disease following therapy, and with progression of disease patient underwent a salvage surgery 8 months later with total laryngectomy, partial pharyngectomy with pectoral major myocutaneous flap reconstruction. Following 3 months post operatively he developed difficulty in swallowing, which progressed to absolute dysphagia at the time of presentation. Multiple attempts at dilatations of the pharyngeal stricture were done, but no respite. Upper gastrointestinal endoscopy revealed a complete stenosis of the pharyngeal stricture with nearly stenosed tracheostoma. Contrast enhanced computed tomography (CECT) neck showed a pharyngeal stricture of approximately $3.5 \mathrm{~cm}$ length starting just below the oropharynx extending from $\mathrm{C} 3$ to $\mathrm{C} 4$ vertebrae (Figure 1).

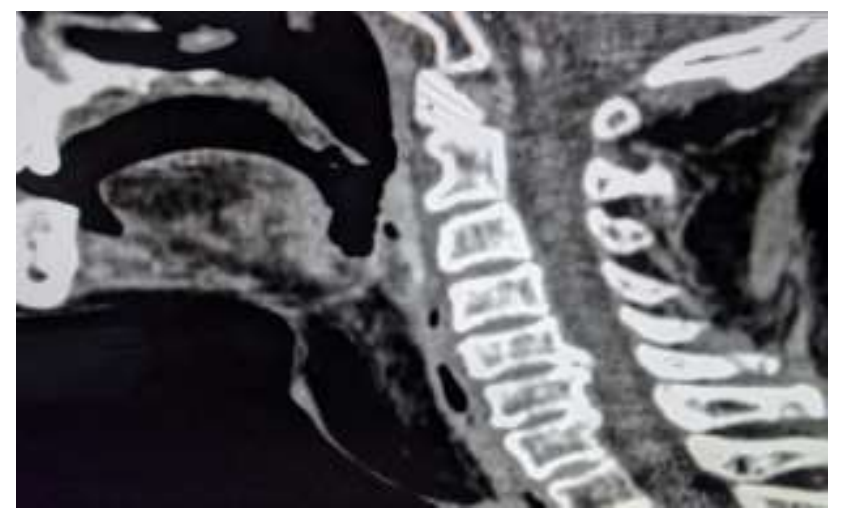

Figure 1: CECT neck showing pharyngeal stricture (3.5 cm in length) opposite $\mathrm{C} 3-\mathrm{C} 4$ vertebrae.

Stenosed tracheostoma was widened by giving two circular and four radial incisions around it (Figure 2). A superior flap was raised along the subcutaneous fat plane up to the tongue base. Pharynx was entered from below, and the stricture seemed involving the neopharynx circumferentially from the base of tongue to oesophagus (Figure 3a). Excision of pharyngeal stricture was performed, leaving a circumferential defect of around $4 \mathrm{~cm}$ (Figure 3b). The pharynx was reconstructed with a pedicled fascio-cutaneous SCAIF, which was used as a tubal conduit between distal oropharynx and proximal esophagus. Pedicle of the flap was marked along with supraclavicular landmarks and vascularity was confirmed using colour Doppler. Flap size approximately $8 \times 4 \mathrm{~cm}$, outline was marked on right side extending posteriorly around $2 \mathrm{~cm}$ anterior to the spine of scapula, and line parallel to posterior line in front of clavicle anteriorly, while lateral margin extended up to the delto-pectoral groove (Figure 3c). Flap was elevated proximally in subfascial plane avoiding damage to the pedicle. At the level of the clavicle plane was changed to subperiosteal to prevent injury to the pedicle (Figure 3d).

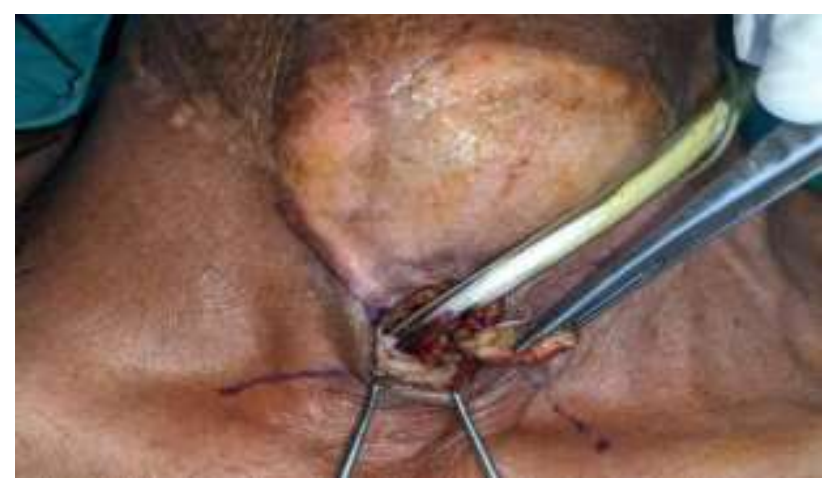

Figure 2: Widening of tracheostoma by two circulars and four radial incisions technique.

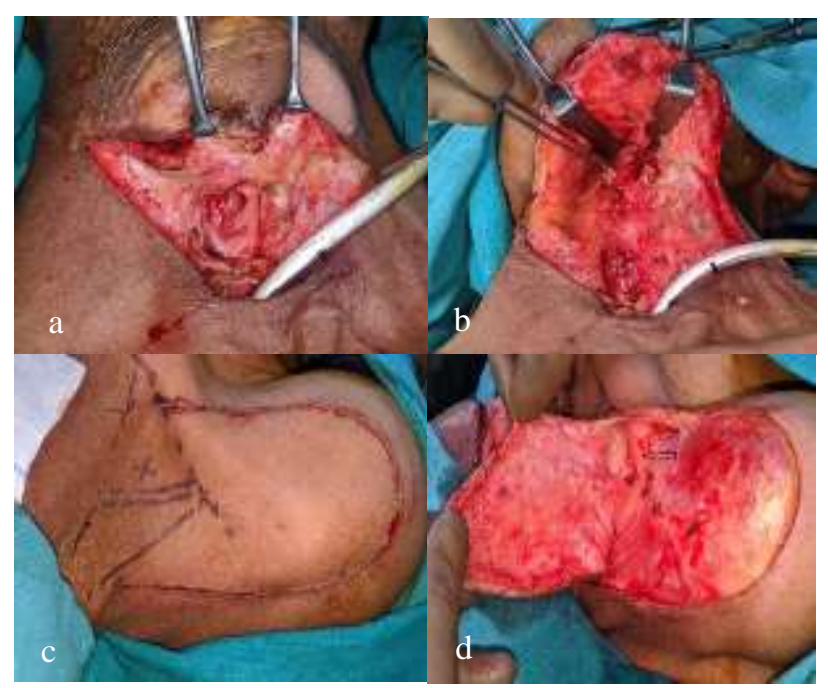

Figure 3: (a) Stricture visualised from below involving the neopharynx circumferentially, (b) excision of the circumferential pharyngeal stricture, (c) markings of the flap based on distal to supra-clavicular landmarks, and (d) elevation of the flap proximally in a sub-fascial plane.

Fully mobilized flap was transposed into the defect; intervening skin was de-epithelized except distal $5 \mathrm{~cm}$ of the flap, which was made as a conduit between distal oropharynx and proximal esophagus over pharyngeal tube (salivary bypass tube $12 \mathrm{~mm}$ ) (Figure 4). Donor site was closed primarily after undermining the flap over the chest and skin over the scapula both anteriorly and posteriorly obviating skin grafting.

On $12^{\text {th }}$ post-operative day, pharyngeal tube was removed and flap was monitored by endoscopy, which demonstrated a healthy flap inset with a conduit patency confirmed with a barium swallow (Figure 5). 
Postoperative results of his swallow study were normal. His diet was progressed to liquid and semisolids over the next several days and adequate functionality of his tracheo-esophageal prosthesis was achieved.

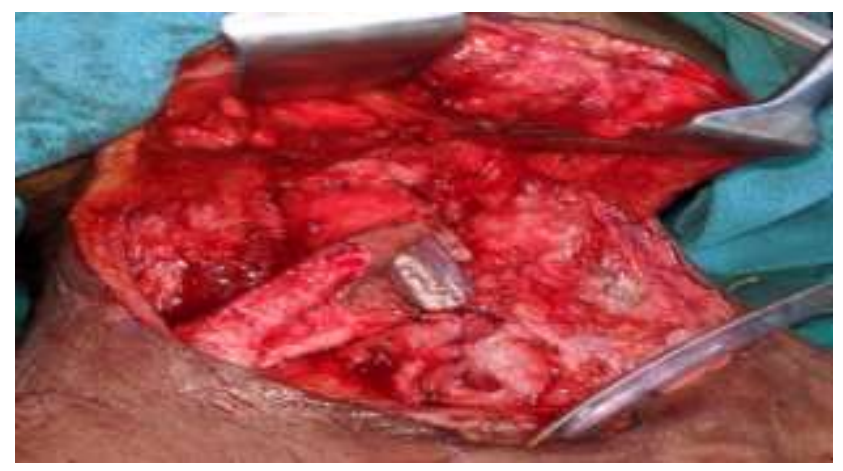

Figure 4: Transposition of the mobilized flap over pharyngeal tube.

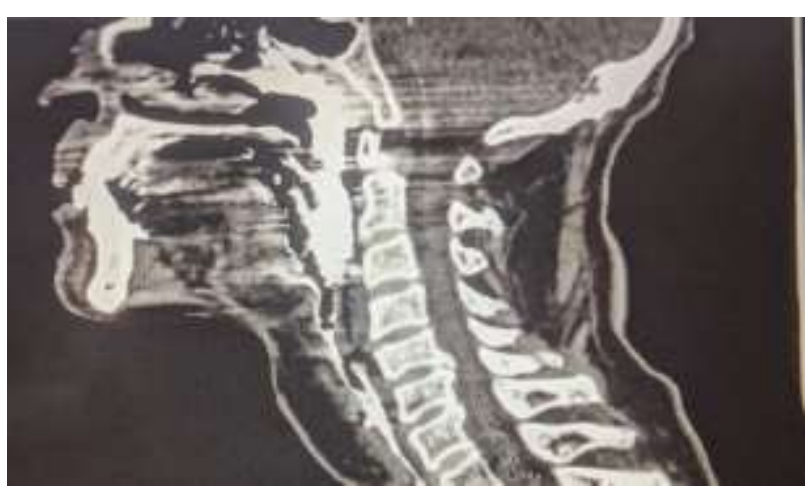

Figure 5: Postoperative barium swallow study day 12 for pharyngeal patency.

\section{DISCUSSION}

Regardless of the wide acceptance of chemo-radiation as a modality for organ preservation for the treatment of initial stages of carcinoma hypopharynx, surgery is still a preferred choice for locally advanced tumors. Reconstruction of circumferential postoperative pharyngeal stricture after tumour extirpation remains a therapeutic challenge. The development of pharyngeal stenosis is a troublesome complication in the late postoperative period after surgery. ${ }^{4}$

Development of swallowing problems in head and neck cancers is a frequent occurrence especially in elderly patients postoperatively. Therefore, senility is considered as a risk factor for pharyngeal stenosis \& stricture in patients with hypopharyngeal cancers treated by concurrent chemo-radiation. Radiation therapy as an independent risk factor may cause a series of problems with swallowing. ${ }^{5}$ The rate of pharyngeal stenosis or stricture is higher among hypopharyngeal cancer patients who received radiation therapy, even when they underwent pharyngolaryngectomy with free flap reconstruction at a later stage. ${ }^{6}$
In our case both the age and previous radiation history are considerable risk factors for development of stenosis due to pharyngeal stricture. Moreover, in order to achieve the best functional and oncological outcome, the choice of the reconstructive options should be individualized according to the patient's characteristics. ${ }^{7}$

An ideal reconstructive choice should not only minimize post-operative complications in an early phase but also aid in long-term gain restoring normal physiology during the post-operative period. The benefits such as reduced donor site morbidity, decreased degree of surgical invasiveness should overweigh the overall risk to the patient. ${ }^{8}$ Myriad of options ranging from laser excision, repeated dilatation, excision of the stricture segment and reconstruction of the hypopharyngeal defect using local cervical flap, pectoral major myocutaneous flap (PMMC), free fasciocutaneous flaps such as the radial forearm or the anterolateral thigh flap, free jejunal interposition and gastric pull-up have been discussed with each having its own advantages and perils. ${ }^{9-15}$

Resection of pharyngeal stenosis with end-to-end anastomosis can be performed in stenosis with limited height. However, in a case of long segment stenosis, resection of the stricture followed by reconstruction with loco regional or free flap gives favorable outcomes.

Patient's profile, poses a dilemma while planning the best reconstructive method in terms of outcome. The patient had undergone partial gastrectomy making him unsuitable for gastric pull-up, while PMMC flap had been used prior for outer skin defect repair during salvage surgery and previous radiation to the neck made the free flap option less dependable. With paucity of viable options it was decided to use a pedicled fascio-cutaneous SCAIF for pharyngeal defect reconstruction, to serve a tubal conduit between the distal oropharynx and the proximal esophagus.

Previous history of neck radiation combined with poor nutrition status precluded microsurgical reconstruction option in this patient. In this context, SCAIF appeared an ideal flap with a thin pliable tissue and superior texture for reconstruction. Although fasciocutaneous flaps based on the supraclavicular artery have been described for several decades its use has recently been popularised among head and neck surgeons. Chiu et al used it for a variety of head and neck oncologic defects. ${ }^{16}$ Liu et al described the use of this flap for functional pharyngeal reconstructions. ${ }^{17}$ Although unilateral SCAIF has already been used in repair of similar defects of smaller dimensions, here we discuss the case where SCAIF is being used for closure of a circumferential pharyngeal defect after resection of the long stricture segment. ${ }^{17,18}$ SCAIF is easily accessible, can be harvested quickly thereby decreasing the operating time significantly. The thin pedicle allows tunneling of the island flap into the defect with considerable ease in the head and neck region and offers a loco regional flap 
alternative with comparable function, results and minimal donor-site morbidity. ${ }^{19}$

\section{CONCLUSION}

Unanimity regarding the best method of reconstructing pharyngeal stenosis following laryngopharyngectomy with minimal related complications and consequences is still debated. It's been seen that in a case of long segment pharyngeal stenosis, resection with reconstruction using a locoregional flap gives a satisfactory outcome. We seek to emphasize by this case report that, in spite of it being the first case report of management of the long segment pharyngeal stricture by resection and reconstruction using supraclavicular flap, it is a versatile flap which is a valuable tool for head and neck defect reconstruction.

Funding: No funding sources

Conflict of interest: None declared

Ethical approval: Not required

\section{REFERENCES}

1. Surkin MI, Lawson W, Biller HF. Analysis of the Methods of Pharyngoesophageal reconstruction. Head Neck Surg. 1984;6(5):953-70.

2. Vu KN, Day TA, Gillespie MB, Harris BM, Sinha D, Stuart RK, et al. Proximal Esophageal Stenosis in Head and Neck Cancer Patients After Total Laryngectomy and Radiation. ORL J Otorhinolaryngol Relat Spec. 2008;70(4):229-35.

3. Kucur C, Durmus K, Ozer E. Supraclavicular artery island flap reconstruction of a contralateral partial laryngeopharyngeal defect. Acta otorhinolaryngol Ital. 2015;35(2):121-4.

4. McConnel FMS, Duck SW, Hester TR. Hypopharyngeal stenosis. Laryngoscope. 1984;94:1162-4.

5. Caudell JJ, Schaner PE, Meredith RF, Locher JL, Nabell LM, Carroll WR et al. Factors associated with long-term dysphagia after definitive radiotherapy for locally advanced head-and-neck cancer. Int J Radiat Oncol Biol Phys. 2009;73(2):410-5.

6. Disa JJ, Pusic AL, Hidalgo DA, Cordeiro PG. Microvascular reconstruction of the hypopharynx: defect, classification, treatment algorithm, and functional outcome based on 165 consecutive cases. Plast Reconstr Surg. 2003;111(2):652-63.

7. Putten VDL, Spasiano R, Bree DR, Bertino G, Leemans CR, Benazzo M. Flap reconstruction of the hypopharynx: a defect oriented approach. Acta Otorhinolaryngol Ital. 2012;32(5):288-96.
8. Couch ME. Laryngopharyngectomy with reconstruction. Otolaryngol Clin North Am 2002;35(5):1097-114.

9. Chu PY, Chang SY. Reconstruction after resection of hypopharyngeal carcinoma: Comparison of the postoperative complications and oncologic results of different methods. Head Neck. 2005;27(10):901-8.

10. Wookey H. The surgical treatment of carcinoma of the hypopharynx and the oesophagus. Br J Surg. 1948;35:249-66.

11. Righini C, Lequeux T, Cuisnier O, Morel N, Reyt E. The pectoralis myofascial flap in pharyngolaryngeal surgery after radiotherapy. Eur Arch Otorhinolaryngol. 2005;262(5):357-61.

12. Azizzadeh B, Yafai S, Rawnsley JD, Abemayor E, Sercarz JA, Calcaterra TC, et al. Radial forearm free flap pharyngoesophageal reconstruction. Laryngoscope. 2001;111(5):807-10.

13. Baek CH, Kim BS, Son YI, Ha Bomjoon. Pharyngoesophageal reconstruction with lateral thigh free flap. Head Neck. 2002;24:975-81.

14. Temam S, Janot F, Germain M, Julieron M, Bretagne E, Myers JN, et al. Functional results with advanced hypopharyngeal carcinoma treated with circular near-total pharyngolaryngectomy and jejunal freeflap repair. Head Neck. 2006;28:8-14.

15. Cahow CE, Sasaki CT. Gastric pull-up reconstruction for pharyngo-laryngoesophagectomy. Arch Surg. 1994;129(4):425-30.

16. Chiu ES, Liu PH, and Friedlander PL. Supraclavicular artery island flap for head and neck oncologic reconstruction: indications, complications, and outcomes. Plast Reconstr Surg. 2009;124(1):115-23.

17. Liu PH, Chiu ES. Supraclavicular artery flap: a new option for pharyngeal reconstruction. Ann Plast Surg. 2009;62(5):497-501.

18. Shenoy A. Supraclavicular artery flap for head and neck oncologic reconstruction: An Emerging Alternative. Int J Surg Oncol. 2013;658989.

19. Pallua N, Machens HG, Rennekampff O, Becker M, Berger A. The fasciocutaneous supraclavicular artery island flap for releasing postburn mentosternal contractures. Plast Reconstr Surg. 1997;99(7):187886.

Cite this article as: Arora V, Kathuria B, Arora M. Technique of neo-pharynx reconstruction with supraclavicular artery island flap in a case of pharyngeal stricture. Int J Otorhinolaryngol Head Neck Surg 2021;7:878-81. 